\title{
KAPAO First Light: the design, construction and operation of a low-cost natural guide star adaptive optics system
}

\author{
Scott A. Severson ${ }^{a}$, Philip I. Choi ${ }^{b}$, Katherine E. Badham ${ }^{a}$, Dalton Bolger ${ }^{b}$, Daniel S. \\ Contreras $^{b}$, Blaine N. Gilbreth ${ }^{a}$, Christian Guerrero ${ }^{c}$, Erik Littleton ${ }^{b}$, Joseph Long ${ }^{b}$, Lorcan P. \\ McGonigle $^{b}$, William A. Morrison ${ }^{b}$, Fernando Ortega ${ }^{b}$, Alex R. Rudy ${ }^{b}$, Jonathan R. Wong ${ }^{b}$ \\ Erik Spjut ${ }^{c}$, Christoph Baranec ${ }^{d}$, and Reed Riddle ${ }^{e}$ \\ ${ }^{a}$ Sonoma State University, 1801 East Cotati Ave., Rohnert Park, CA 94928, USA; \\ ${ }^{b}$ Pomona College, 610 North College Ave., Claremont, CA 91711, USA; \\ ${ }^{c}$ Harvey Mudd College, 301 Platt Boulevard, Claremont, CA 91711, USA; \\ ${ }^{d}$ Institute for Astronomy, University of Hawaii, 640 North A'ohoku Place, Hilo, HI 96720, USA; \\ ${ }^{3}$ California Institute of Technology, 1200 E. California Blvd., Pasadena, CA, 91125, USA
}

\begin{abstract}
We present the instrument design and first light observations of KAPAO, a natural guide star adaptive optics (AO) system for the Pomona College Table Mountain Observatory (TMO) 1-meter telescope. The KAPAO system has dual science channels with visible and near-infrared cameras, a Shack-Hartmann wavefront sensor, and a commercially available 140-actuator MEMS deformable mirror. The pupil relays are two pairs of custom off-axis parabolas and the control system is based on a version of the Robo-AO control software. The AO system and telescope are remotely operable, and KAPAO is designed to share the Cassegrain focus with the existing TMO polarimeter. We discuss the extensive integration of undergraduate students in the program including the multiple senior theses/capstones and summer assistantships amongst our partner institutions. This material is based upon work supported by the National Science Foundation under Grant No. 0960343.
\end{abstract}

Keywords: Adaptive optics, astronomical adaptive optics, visible light adaptive optics, MEMS, dual-band imaging, undergraduate research mentoring

\section{INTRODUCTION}

The KAPAO instrument represents the culmination of a multi-institution partnership to develop, build and deploy a low-cost, remote-access, natural guide star adaptive optics system for the Pomona College Table Mountain Observatory (TMO) 1-m telescope. This micro-electro-mechanical systems (MEMS) deformable mirror (DM) based astronomical AO system is capable of simultaneous dual-band, diffraction-limited imaging at optical and near-infrared wavelengths. The project consists of a unique collaboration of private (Pomona College, PC, and Harvey Mudd College, HMC) and public (Sonoma State University, SSU) undergraduate institutions designing and building the system with the essential control software provided by our research university partner, California Institute of Technology (CIT), and their Robo- $\mathrm{AO}^{1}$ partner, the Inter-University Centre for Astronomy and Astrophysics. A discussion of the integral efforts of undergraduate student researchers can be found in Section 5 . Our collaboration produced a prototype system, KAPAO-Alpha, ${ }^{2}$ which informed our design for the facility instrument, KAPAO-Prime (henceforth simply KAPAO). We present the design and early results of KAPAO, the facility class instrument that saw First Light in the second half of 2013.

The project goals of KAPAO are as follows. First, add high-resolution imaging capabilities to TMO, expanding current science programs with a focus on high-resolution, time-domain astrophysics. Second, introduce $\mathrm{AO}$ technology and techniques to a broad range of students; train a generation of undergraduates in both astronomical AO research and instrument design; and fill future AO science and engineering pipelines. Third,

Further author information:

S.A.S.: E-mail: scott.severson@sonoma.edu, Telephone: 17076642376

P.I.C.: E-mail: philip.choi@pomona.edu, Telephone: 19096070890

Adaptive Optics Systems IV, edited by Enrico Marchetti, Laird M. Close,

Jean-Pierre Véran, Proc. of SPIE Vol. 9148, 914839 - () 2014 SPIE

CCC code: $0277-786 \mathrm{X} / 14 / \$ 18 \cdot$ doi: $10.1117 / 12.2056961$ 
to serve as a development platform, using the completed facility-class AO instrument as an on-sky testbed for future undergraduates to explore expanded capabilities (e.g., coronagraphy, polarimetry and advanced wavefront sensing technologies).

\section{KAPAO'S BEGINNINGS}

The KAPAO instrument we describe in this paper is related to two other AO instruments for use on modest aperture telescopes: ViLLaGES and RoboAO. The Villages visible light AO system ${ }^{3-6}$ was a forerunner in using a MEMS Deformable Mirror. The Robo-AO ${ }^{1,7,8}$ autonomous laser guide star AO system is a recently completed MEMS DM based system and is the source for the KAPAO AO loop control software. ${ }^{9}$ These systems are based on the same DM architecture as KAPAO, a 140-actuator BMC MEMS deformable mirror. ${ }^{10}$ For a treatment of matters relating to the use of MEMS devices for astronomical AO use, see Morzinski 2012. ${ }^{11}$

The KAPAO consortium conceived a rapid-deployment prototype, KAPAO-Alpha, once we had early success in adapting the Robo-AO control software to our laboratory testbed system. Based on the same MEMS DM and SciMeasure E2V-CCD39 wavefront sensor (WFS) as the Robo-AO system, we were able to close the AO loop with an in-lab testbed in summer 2011. With key questions regarding the mechanical coupling and software integration with the telescope as well as a preference to get students working on these issues early, we split the development of KAPAO into the rapid design, construction and use of the simpler KAPAO-Alpha prototype, ${ }^{2}$ while taking time to build the final KAPAO instrument on our original timetable.

For KAPAO-Alpha, we did not include a near-infrared camera leg and used commercially-available OAPs which required a lens-based pupil expansion relay. Despite these sacrifices, we: closed the loop on sky with this system in 2012; solved mechanical and cable routing issues; and gained confidence with our mounting solution. KAPAO-Alpha and the final KAPAO system were designed with a 1-meter square optical breadboard plate forming the basis of the instrument. Unlike ViLLaGES, KAPAO-Alpha and KAPAO were designed without a spaceframe mount between the $\mathrm{AO}$ table and the telescope. Instead, in order to minimize the space taken up before the telescope back focal plane, we went with an "upside-down" mounting scheme that placed the breadboard directly against the telescope primary mirror mount. We used a $45^{\circ}$ fold mirror to bring the beam to the optical plane of the board. Our experience with KAPAO-Alpha was critical in verifying this solution and was used in the final KAPAO design.

\section{KAPAO'S DESIGN}

Figure 1 presents a CAD render of the KAPAO optical table and serves as an overview of the design. The instrument optical path begins with the Fold Mirror and shortly thereafter is the Cassegrain focus of the telescope (where we have subsequently inserted a white-light and red diode laser reference source stage, see Figure 4). Following our first custom Off Axis Parabola (OAP1) we form a pupil at the Physik Instrumente piezo tiptilt mirror (TT). Then a relay (OAP2 and OAP3) places the pupil on our 140-actuator BMC MEMS DM. OAP4 follows, where the now-converging beam encounters the first dichroic beamsplitter (BS1). The reflection wavelengths (200-686 nm) head to our WFS relay where we have constructed a Shack-Hartmann WFS that uses a SciMeasure E2V-CCD39 camera. The transmission wavelengths of BS1 (710-1650 nm) proceed to a second dichroic beamsplitter (BS2). There, short wavelengths are sent to our Andor iXon EMCCD optical camera (710-1000 nm) by way of a fold mirror and relay lens. Wavelengths longer than 1 micron are imaged directly on our Xenics Xeva-1.7-320 near-infrared camera (sensitive from 0.9 to 1.7 microns). This configuration allows simultaneous dual-band (optical and near-infrared) imaging. Both cameras are capable of operating at high framerates and we have used this capability to demonstrate improved performance through frame selection (see Section 4).

KAPAO uses custom designed OAPs to simplify the optical design. The static optical system produces diffraction limited images over a substantial $80^{\prime \prime}$ diameter field of view. The optical design places the telescope pupil on the tip-tilt mirror, and on the $4.4 \mathrm{~mm}$ x $4.4 \mathrm{~mm}$ MEMS DM. OAP4 produces an f/46.4 (0.133 "/pixel) beam on the near-infrared Xenics Xeva-1.7-320 camera (30 micron pixels in a 320x256 array). After a relay, the Andor iXon3 888 optical camera (13 micron pixels in a 1024x1024 array) sees an f/35.2 beam (0.076 "/pixel). For comparison, at TMO the $\mathrm{z}^{\prime}=905 \mathrm{~nm}, \mathrm{~J}=1.25$ micron, and $\mathrm{H}=1.65$ micron bandpasses have $\lambda / D$ of: $0.187^{\prime \prime}$, 


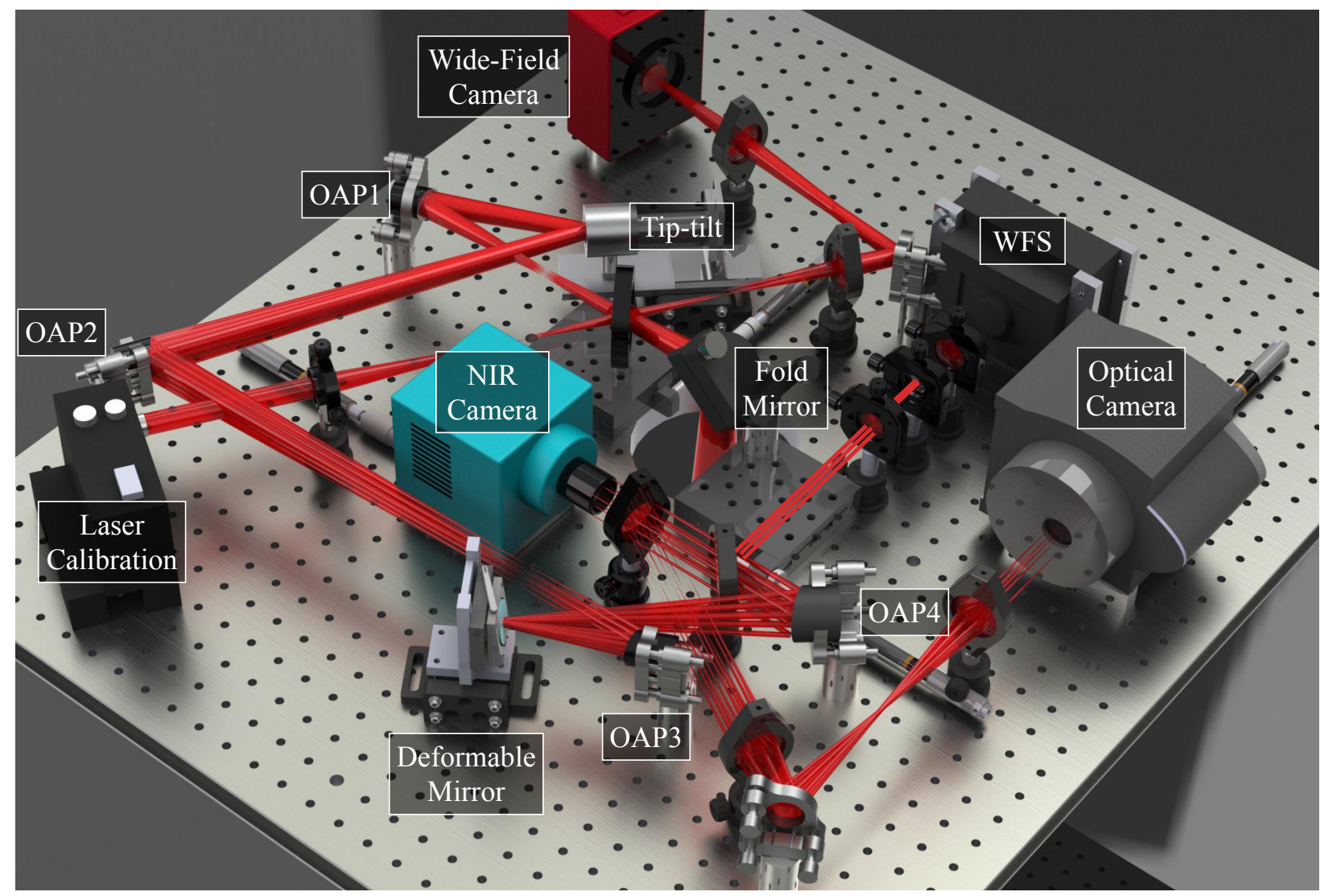

Figure 1. A CAD render of the KAPAO instrument design, showing the central fold mirror directing the telescope beam (red) to the custom OAP mirrors, the tip-tilt mirror, the MEMS deformable mirror, the wavefront sensor, and science cameras (nir and optical). Visible in the figure, but left unlabeled for clarity are the two dichroic beamsplitters that serve to direct the beam to the WFS, the nir camera, and the optical camera. A laser alignment source and wide-field camera, evident in the CAD, have been replaced in the as-built instrument (see Figure 4).

$0.258^{\prime \prime}$, and $0.340^{\prime \prime}$, respectively. Fabricated to tolerance and aligned, this OAP relay does not contribute any recognizable wavefront error for an on-axis source. For the four corner field positions on the wider-field optical camera $\left(+/-40^{\prime \prime}\right)$, the average wavefront error contribution was $30 \mathrm{~nm}$. The average corner RMS spot radius was 14.6 microns, which compares favorably with the Airy Disk radius of 34.0 microns. These results are shown in Figure 2, which includes both the spot diagrams and encircled energy curves.

Two other KAPAO components bear further discussion. KAPAO upgraded the previous tip-tilt mirror (TT) from that used in KAPAO-Alpha. The closed-loop tip-tilt angle increased from 2 mrad to $10 \mathrm{mrad}$. We encountered a resulting issue that the new 3" TT barrel sags when the KAPAO instrument is flipped upside down and mounted on the telescope. We reinforced the TT mirror mounting with a larger metal barrel that acts as a kinematic guide. Another upgrade, the inclusion of a white light source (WLS) increases the alignment capabilities to a dual calibration system. Previously, only a $635 \mathrm{~nm}$, variable-power red laser was present in the system. The optical path passes through a $700 \mathrm{~nm}$ High Performance Longpass Filter on the way to the science cameras. Now with the WLS, we may align the optical science, near-infrared science and wavefront sensor legs simultaneously.

KAPAO is designed to be operated remotely, which is a standard observing mode at TMO. In addition, KAPAO shares the Cassegrain focus with the TMO Polarimeter. KAPAO includes a fold mirror on a linear stage to intercept the beam or to allow the entire field to pass to the polarimeter. Several other stages are motorized for remote control: the light-source calibration stage that inserts at the telescope focus; the optical 

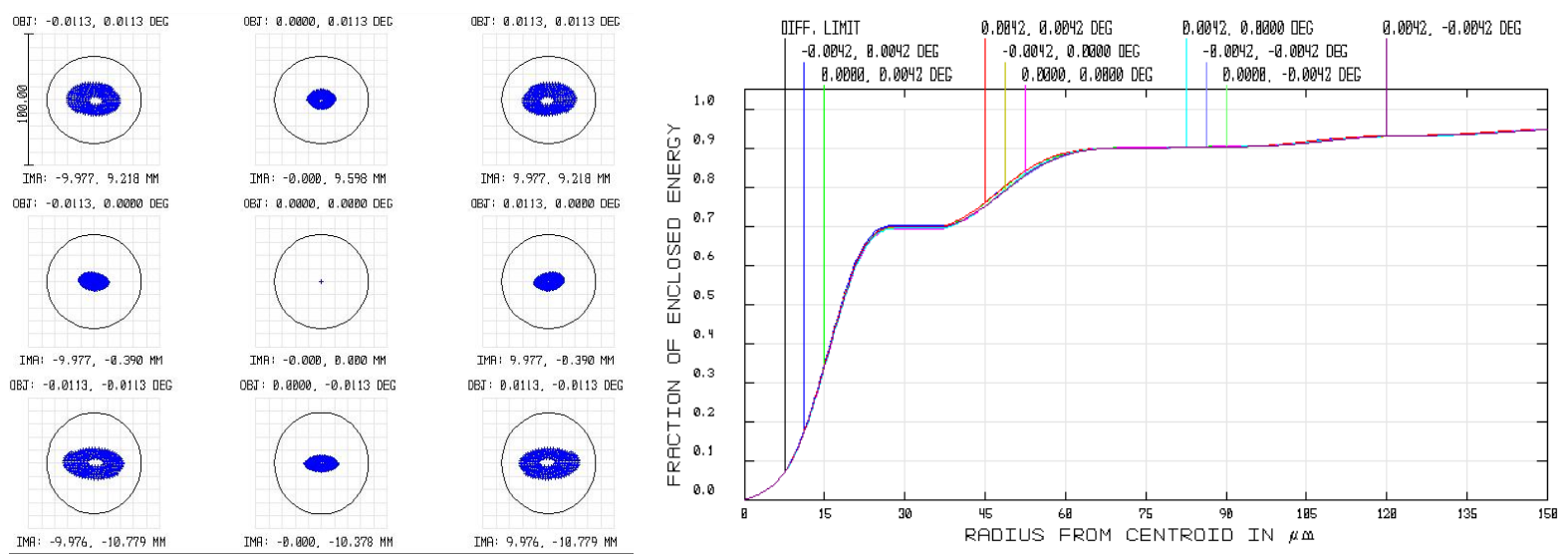

Figure 2. KAPAO spot diagrams and encircled energy curves spanning a $80^{\prime \prime} \mathrm{x} 80^{\prime \prime}$ field-of-view. The spot diagrams [left] show that geometric aberrations due to our optical design are negligible compared to the diffraction limit, represented by the Airy disk (black circle). FFT diffraction enclosed energy curves [right] are indistinguishable from the diffraction limit over the central $80^{\prime \prime} \times 80^{\prime \prime} \mathrm{FOV}$ of the KAPAO optical camera.

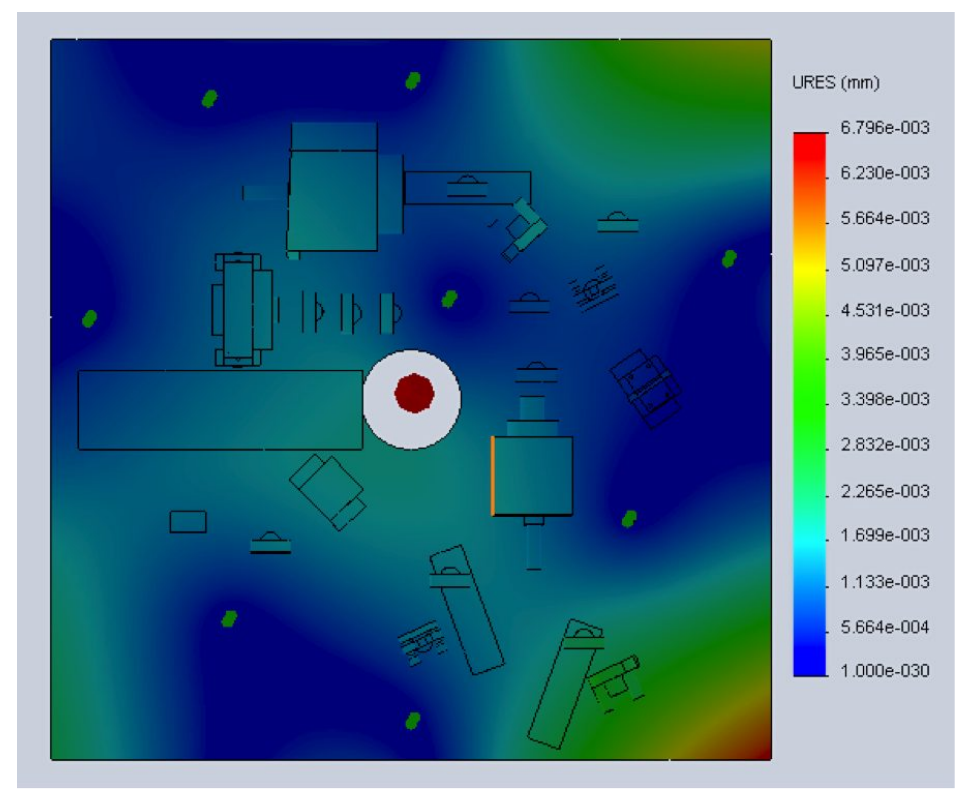

Figure 3. Finite element analysis of the KAPAO optical breadboard deflection. In the run visualized here, gravity is normal to the plane of the board. The small green spots show the location of the mounting bolt holes. The color scale shows deflections from 0 to 7 microns. With a flexure of no more than 6 microns (and typically less that 3 microns) which results in a deflection of less than $1.5 \%$ of an actuator at the MEMS DM, the "upside-down" breadboard mounting scheme has proven successful.

and near-infrared camera focus stages; the six-position optical filter wheel; and the near-infrared filter flip-mount. The DM control electronics are mounted directly to the optical bench, several other controllers such as the camera electronics are mounted in a separate box attached to the telescope, and the rest of the control systems are in a cart attached via an umbilical cable bundle.

We had experience in addressing telescope mounting issues and flexure concerns with KAPAO-Alpha as mentioned in Section 2. Our OAPs are aligned to a precision of less than 10 microns in any direction, the approximate limit of our capabilities. This corresponds to less than $1 \mathrm{~nm}$ of wavefront error when applied as 


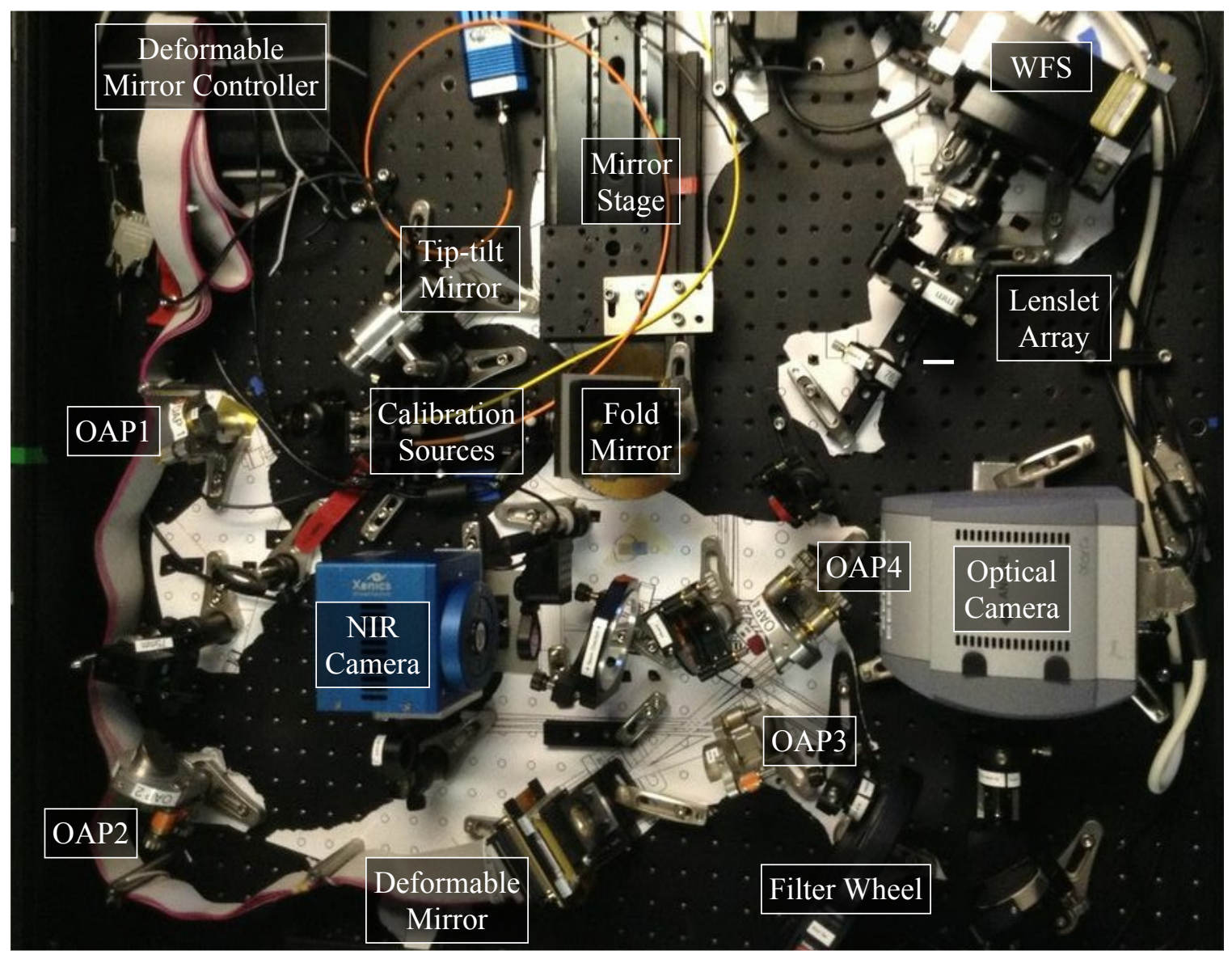

Figure 4. A detailed view of the KAPAO instrument, as-built. The optical bench is mounted to the telescope upside down, so in this view we are looking up at the board mounted underneath the primary. The Cassegrain focus is folded into a plane parallel to the bench at a 3" height from the board. The OAP relay and key components (TT, DM, WFS, and science cameras) are evident. In this figure, there are several items not shown in the CAD (see Figure 1): the linear stage for moving the fold mirror out of the way and allowing the telescope beam to pass to a polarimetric camera that shares the Cassegrain focus; the white-light and red laser calibration sources; the optical filter wheel and NIR filter flip mount; and the DM controller. The use of a 1:1 scale print-out of the layout to assist in placement of optics is apparent as the white cut-outs remaining on the board.

decenter tolerances on the OAPs for KAPAO. The optical breadboard supporting the OAPs must also be rigid to these tolerances. We designed and modeled the opto-mechanics of KAPAO in SolidWorks 3D. We produced a detailed simulation of the honeycomb breadboard's flexure due to gravity and deformation due to mounting and temperature changes. These simulations then informed a redesign of the system's mounting holes and the cage that protects the system and supports the TMO Polarimeter instrument. Figure 3 shows a Finite Element Analysis (FEA) of the resulting flexure of KAPAO. Our experience with KAPAO-Alpha FEA and its fine mechanical performance gave us great confidence with this solution and we have had no issues with the final KAPAO.

The predicted performance of the system including limiting magnitude and strehl performance is discussed in our KAPAO-Alpha paper. ${ }^{2}$ The results of Yao Monte-Carlo simulations ${ }^{12}$ suggested that the system is capable at both optical and near-infrared wavelengths. For example, with typical seeing at TMO of about $1.5^{\prime \prime}$, we calculate a limiting NGS magnitude of 10th magnitude for moderate (Strehl=0.4) H-band correction. We discuss actual closed-loop performance in the next section, but it is important to note that the results presented in this paper are with the system without the high reflectivity protected silver coatings we had applied recently in 2014. Without 


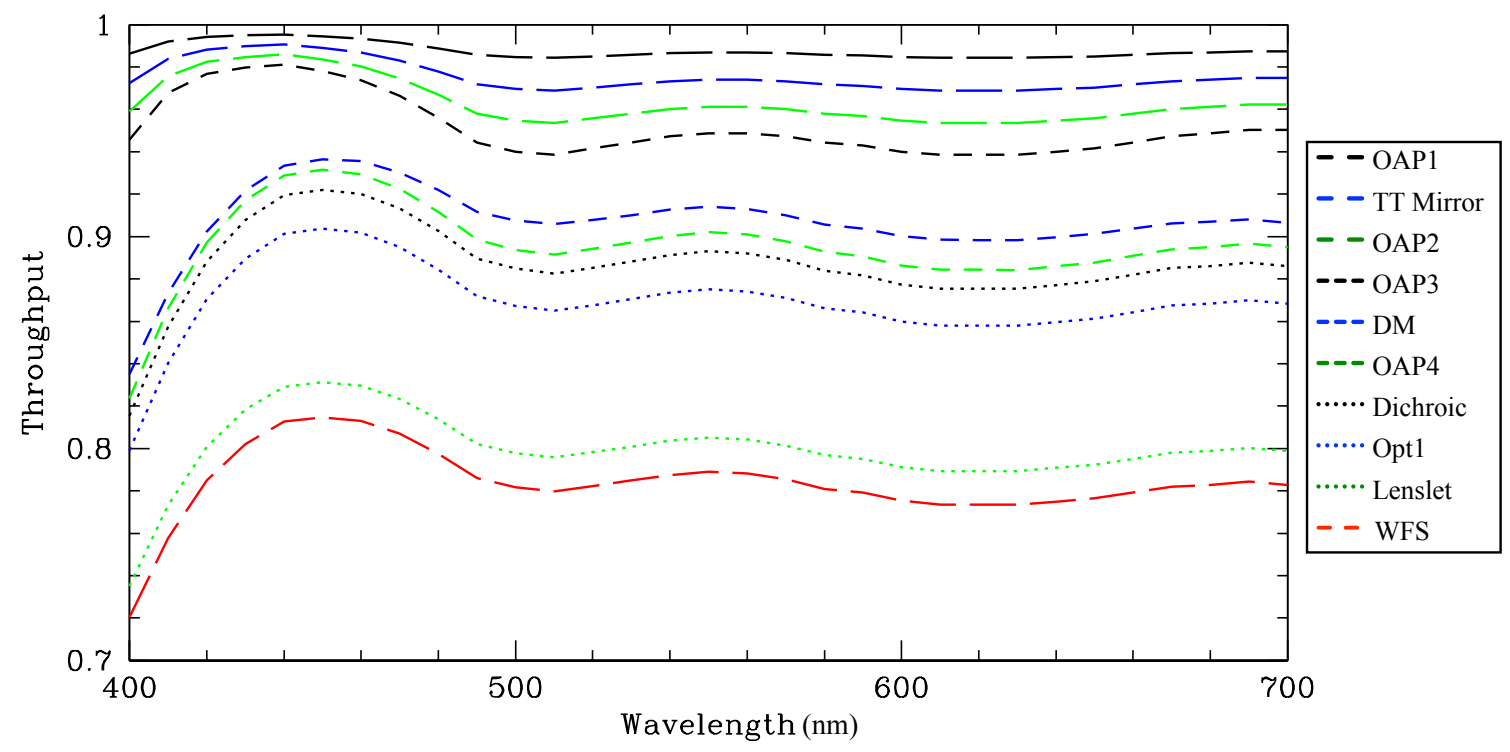

Figure 5. Theoretical throughput for the KAPAO system (excluding telescope), component by component, to the WFS. The values used for each component are taken from the reported values of the respective manufacturers for the wavelength range of interest for the WFS.

these coatings in place, our presented measurements do not address the system throughput and observing limits. We have addressed this by preparing a theoretical throughput for the KAPAO system by using a component by component model. This is presented in Figure 5, and shows that with our new coatings we can expect an instrument throughput of greater than $75 \%$ from Cassegrain focus to wavefront sensor.

\section{KAPAO'S FIRST LIGHT}

We are presenting results representing two runs from the second half of 2013 (see Table 1). A late July run culminated the summer undergraduate research session. The July observations were intended to validate the summer engineering work and get initial observations with closed-loop AO correction. After a Fall semester of work integrating many of the automation components and the near-infrared camera, we returned to TMO for a second run. Both of these runs were done with uncoated OAP mirrors, and hence we present no throughput analysis. Figure 6 shows the KAPAO system mounted on the TMO 1-meter telescope. The mounting of the KAPAO optics table to the telescope consists of a flip of the instrument followed by a guided lift onto fiducial mounting pins. The system has made several trips between the Pomona College campus and the telescope and has proven to be robust and portable.

Table 1. An overview of performance parameters during the KAPAO runs discussed in the paper. For both the uncorrected and AO-corrected data we present an RMS wavefront error calculated from wavefront sensor telemetry, and a Strehl ratio calculated from co-added images. The July data was optical only and at the entire wavelength range (710-1000 nm) passed by the dichroic. The December data includes the NIR H' and Sloan z' bands. The July data is of $\beta$ Pegasi (V=2.42). The December data is of $\eta$ Piscium ( $\mathrm{V}=3.62)$.

\begin{tabular}{cccccc}
\hline Observation & \multicolumn{2}{c}{ Uncorrected } & \multicolumn{2}{c}{ AO-corrected } \\
Period & Filter & RMS (nm) & Strehl & RMS (nm) & Strehl \\
\hline July 2013 On-sky & Optical & 220 & 0.08 & 60 & 0.26 \\
\hline Dec 2013 On-sky & NIR H' & 180 & 0.138 & 50 & 0.436 \\
\hline Dec 2013 On-sky & Sloan z' & 180 & 0.051 & 50 & 0.294
\end{tabular}




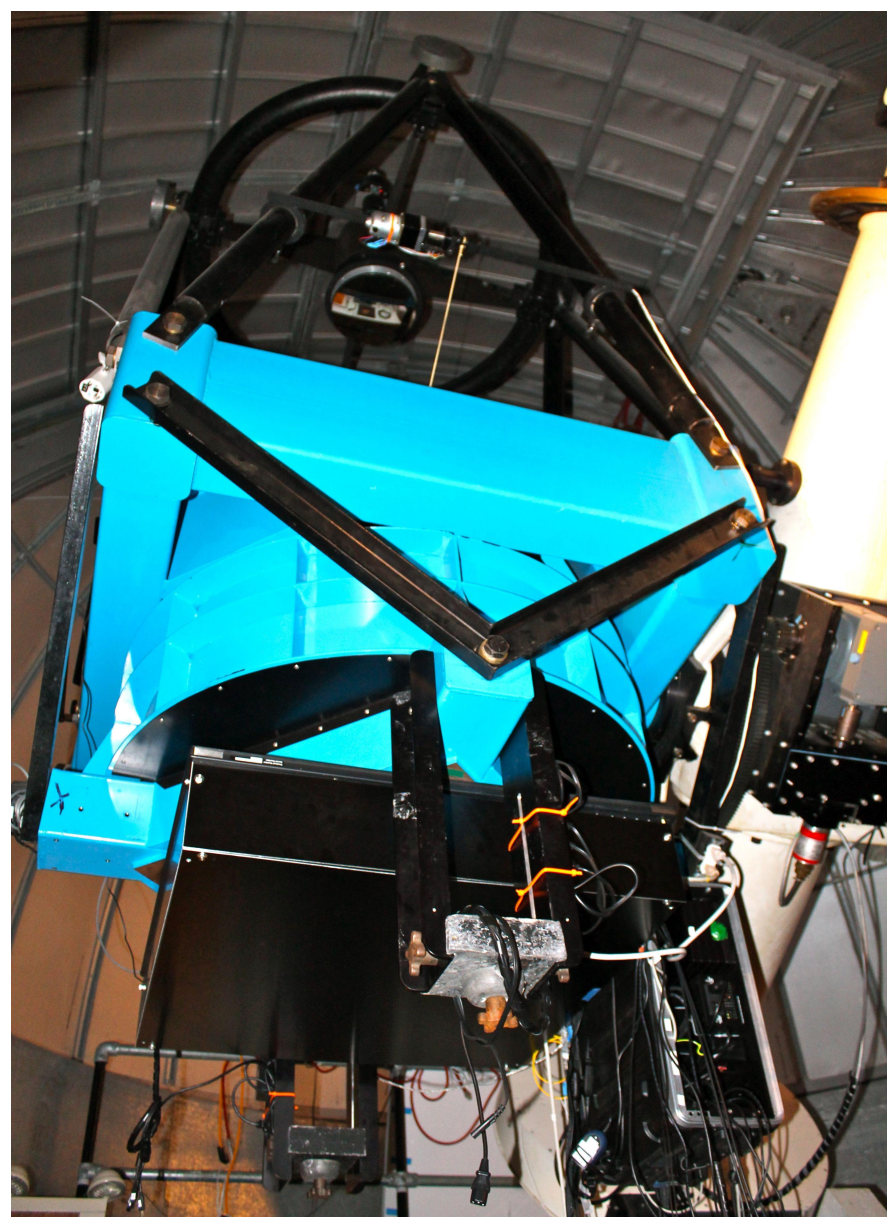

Figure 6. KAPAO on the Table Mountain Observatory 1-meter telescope during the December 2013 on-sky run. The KAPAO optical bench is enclosed in black anodized panels and is shown mounted underneath the telescope (blue) and cabled to the MEMS DM and camera controllers, which are further cabled to the Loop Control computer running the Robo-AO software under Linux and the Camera Control computer running our science and alignment cameras under Windows. The whole system is mounted onto the telescope with the optics hanging upside-down compared to the lab configuration.

Figure 7 (Sloan $z^{\prime}$-band) and Figure 8 (H-band near-infrared) show open and closed-loop images on the star $\eta$ Piscium $(\mathrm{V}=3.62)$, during atmospheric seeing of $\approx 1^{\prime \prime}$. These simultaneous observations are from the December run, and show the dramatic image improvement with KAPAO.

\subsection{LUCKY IMAGING}

During these inaugural observations with KAPAO, our dual science cameras were configured to take images with very short integration times. This gave us frames short enough to freeze the atmosphere and allowed us to add another layer of analysis, that of "lucky imaging" $13 .{ }^{14}$ In short, "lucky imaging" is frame-selecting images with residual wavefront error (and hence Strehl) at some value better than the median. We performed postprocessing on images that combined "lucky imaging" techniques with AO correction. By splitting the data cubes and sorting frames by their peak flux values, a simple cutoff can be employed to take only the $N$ luckiest images from a particular observation. These lucky frames are then shifted and co-added, correcting any residual tip-tilt error. In the text and figures, when we refer to "AO lucky" frames, we mean frames processed in this manner. Note: the vast majority of the improvement in our images is due to the AO system, not the post-processing (see below). However, it is interesting to note that AO lucky processing provides greater improvement to the Sloan $z^{\prime}$ 


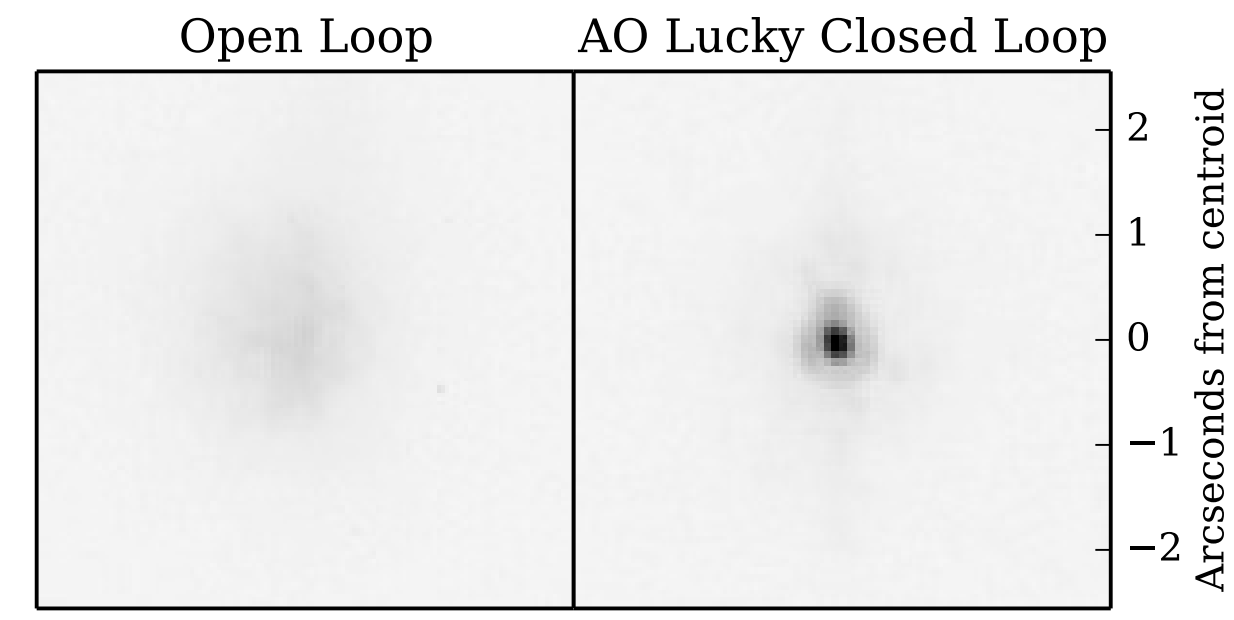

Figure 7. Optical Sloan $z^{\prime}$ band side-by-side comparison of open/seeing-limited and AO lucky (see Section 4.1) images of $\eta$ Piscium.

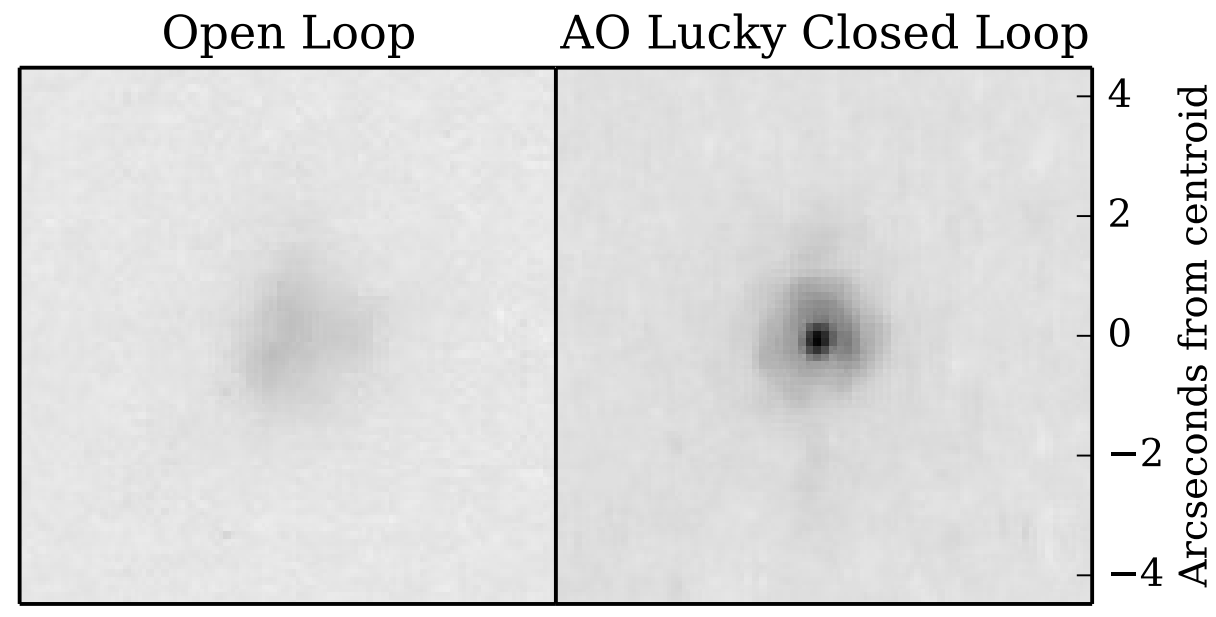

Figure 8. Near-IR H-band side-by-side comparison of open/seeing-limited and AO lucky (see Section 4.1) images of $\eta$ Piscium.

exposures, despite the overall lower Strehl. Nonetheless, Strehl ratios for both optical and near-infrared images improve with the AO lucky processing.

\subsection{CURVE OF GROWTH}

We calculated curves of growth for our near-infrared and optical band images to detail the structure of our Point Spread Functions (PSFs). Using different subsets of frames from our observations, we can compare open-loop, closed-loop, and AO lucky PSFs with an ideal PSF. The "Ideal PSF" is constructed from a zero-aberration simulation using our telescope pupil. The "Open" image is the construction of a seeing limited observation by co-adding the uncorrected frames from an image data cube. The "AO Closed" image is a single AO-corrected frame. And the "AO Lucky" image is constructed from the $N$ luckiest images from a particular observation, shifted and co-added. We present the results of this analysis in Figure 9 and Figure 10. Notable are the expected improvement from the AO system in closed loop, the incremental improvement when using lucky image processing, and the peak AO Lucky Strehls of over 0.4 in both the H-band and the Sloan $z^{\prime}$ filter. 


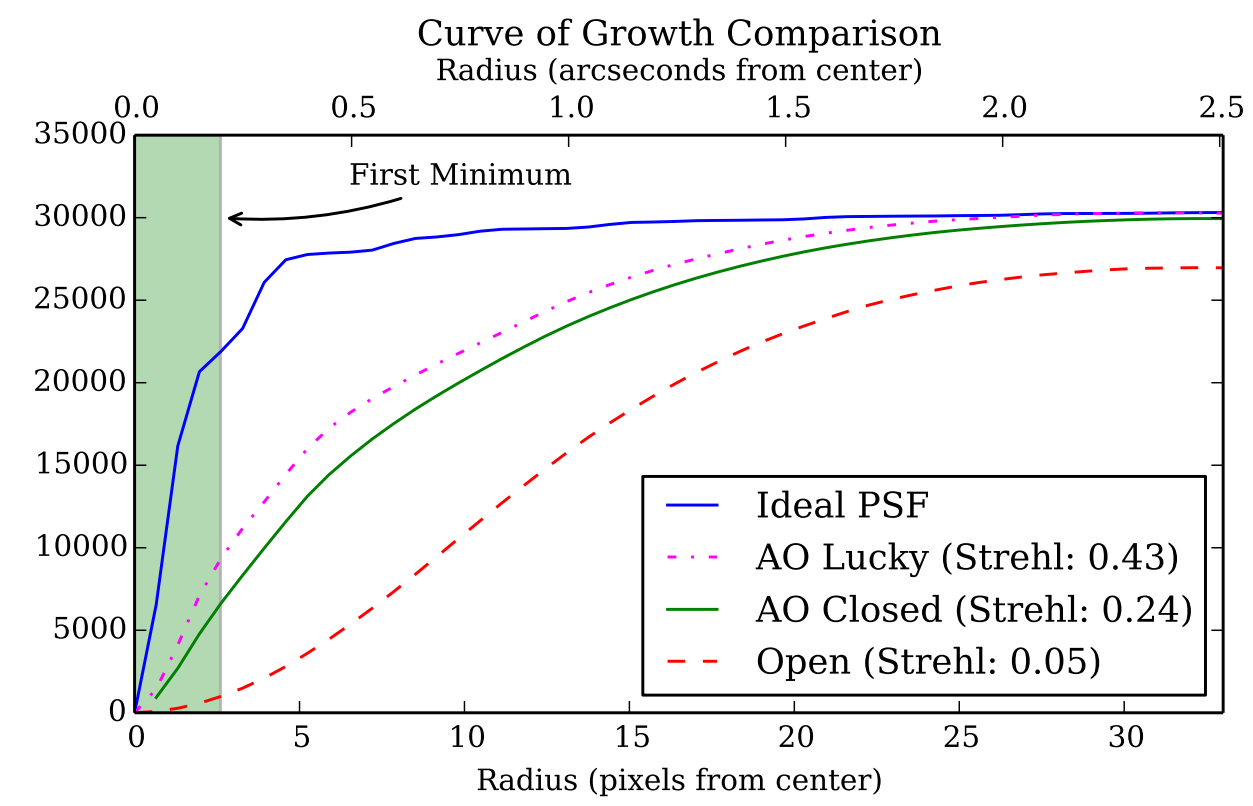

Figure 9. Curve of growth comparison for Sloan $z^{\prime}-$ band images of $\eta$ Piscium. Included are the curves of growth for: an ideal PSF, an open loop/seeing-limited frame, a typical closed loop frame, and an AO lucky frame.

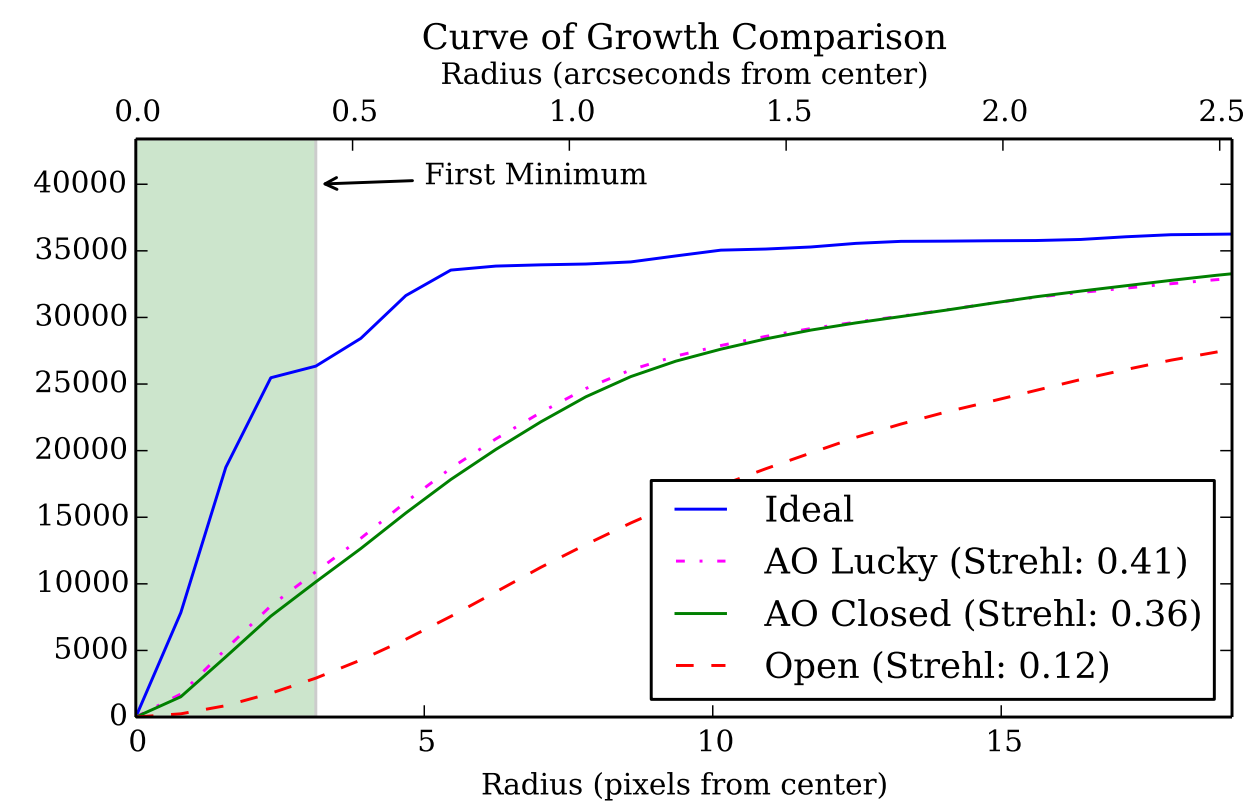

Figure 10. Curve of growth comparison for H-band images of $\eta$ Piscium. Included are the curves of growth for: an ideal PSF, an open loop/seeing-limited frame, a typical closed loop frame, and an AO lucky frame.

\subsection{STREHL}

We use the Strehl values we calculate from our $\eta$ Piscium data cubes to characterize instrument performance. A frame-by-frame Strehl ratio time-series gives an idea of how AO-corrected performance compares to both open-loop operation (i.e. seeing-limited observation) and lucky imaging, where one uses short integration times to freeze turbulence but not AO correction. Figure 11 and Figure 12 plot Strehl values over time, clearly showing 
the point where KAPAO AO correction begins. As in the curve of growth analysis, the open loop Strehl ratios noted on the plots are obtained by analyzing a co-added image made from the frames taken without any AO correction. The speckle pattern mean Strehl is the mean instantaneous Strehl measurement from individual frames taken without AO correction. The noted AO closed loop mean Strehl is simply the mean over those frames taken after the loop closes. The AO Lucky strehl for each run is higher than those shown within these plots, and comes from the frame selection we have discussed.

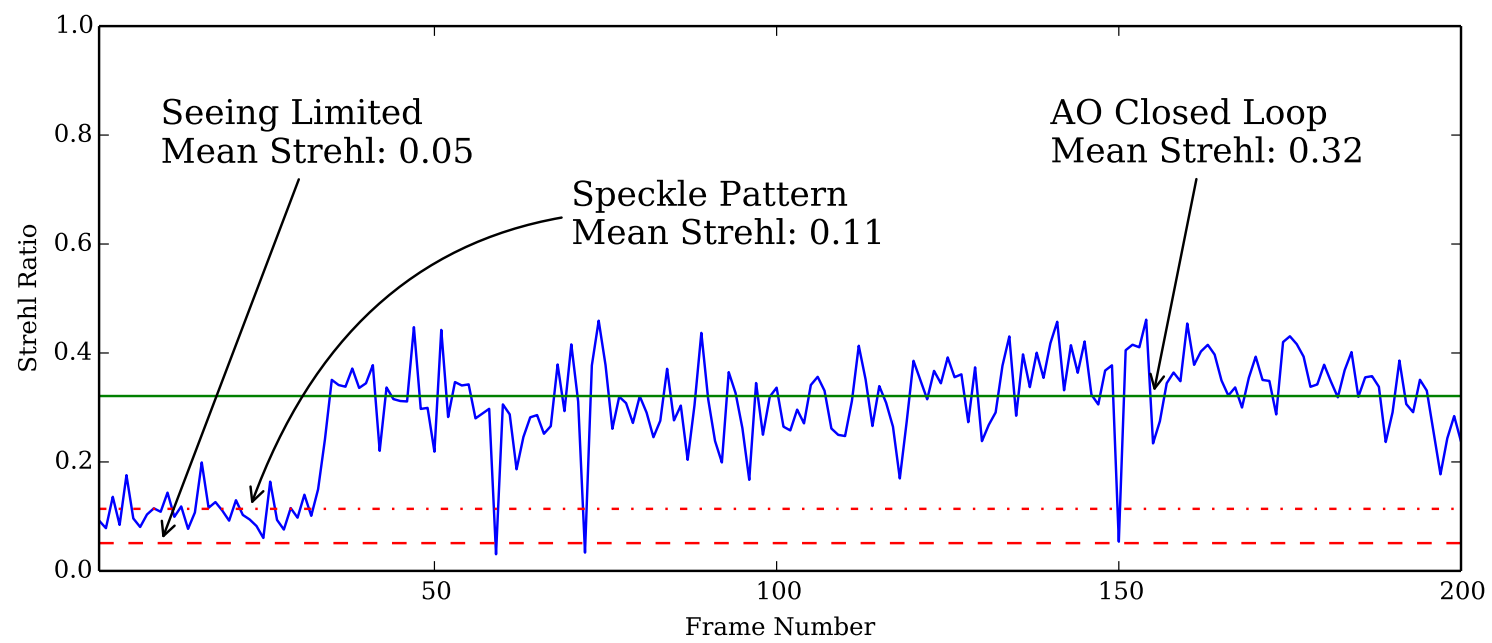

Figure 11. Plot of optical (Sloan $z^{\prime}$ band) Strehl ratios computed frame-by-frame for observations of $\eta$ Piscium. Integration time was 0.05 seconds per frame.

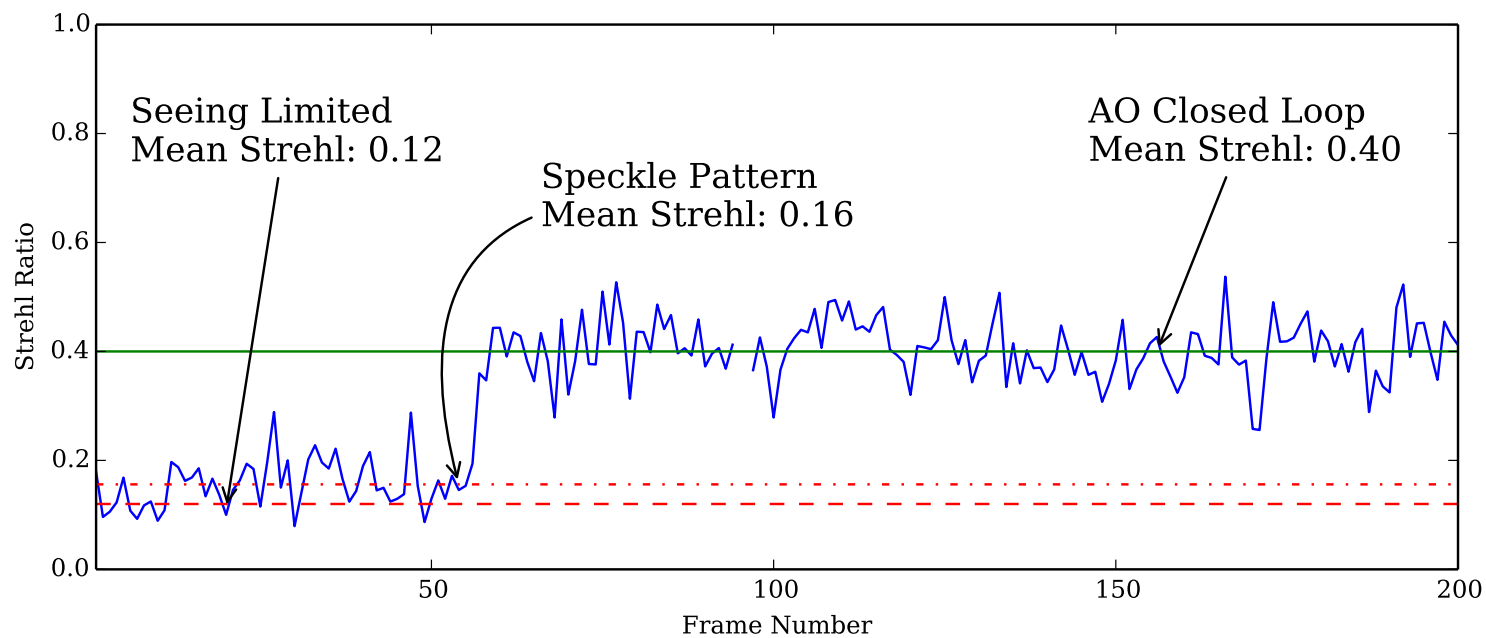

Figure 12. Plot of near-IR (H-band) Strehl ratios computed frame-by-frame for observations of $\eta$ Piscium. Integration time was 0.02 seconds per frame.

\section{UNDERGRADUATE STUDENT EFFORT}

The inclusion of undergraduate student work in all aspects of this effort is integral to our process. The work presented herein includes significant contributions from our undergraduate students within the framework of a carefully structured research environment. Fifteen different undergraduate students have made significant 
contributions over the course of the project. Student involvement has come in the form of both summer and year-long research internships and has produced eight senior research theses, was well as many posters and talks. The bulk of the summer work has been hosted at Pomona College, with students from Harvey Mudd College and Sonoma State University joining the effort. Students have had specific responsibilities in the project as denoted in Figure 13. For a discussion of the design of this sort of undergraduate research see Severson $2010 .{ }^{15}$ Of the ten students who have graduated so far, five have gone onto doctoral programs in physics, astrophysics, aerospace engineering, electrical engineering, and computer science and three more have gone directly into engineering industry positions. It is important to stress that undergraduate student effort is key to all areas of the success of the KAPAO instrument.

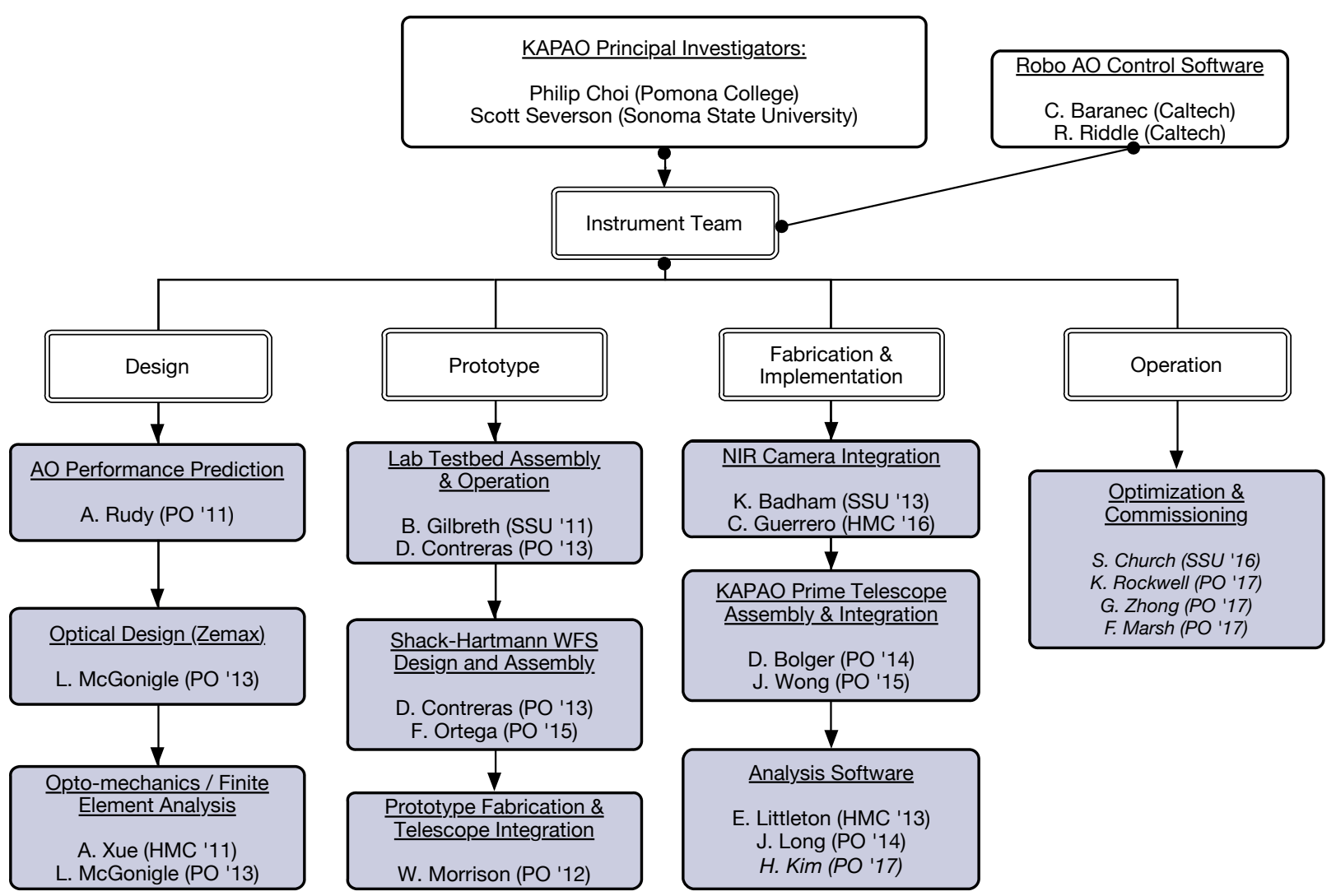

Figure 13. An organization chart of the KAPAO project displays the emphasis on undergraduate student effort and training at the heart of the project. Each shaded project areas contain a list of the students responsible for this portion of KAPAO. They are listed with their institution and year of graduation. Current KAPAO students are shown in italics.

\section{FUTURE PLANS}

KAPAO is a brand new facility AO instrument for the TMO 1-meter telescope. The recently applied high-quality protected silver coatings will increase system throughput and place many bright time-domain astronomical targets within our reach. The system will continue to serve as a source of undergraduate training in adaptive optics, with continued authentic astronomical and instrumental projects (including image sharpening to reduce noncommon path aberrations). Finally, the further development of the system and the site to provide remote access to KAPAO will broaden the impact of the system throughout the KAPAO collaboration. 


\section{CONCLUSIONS}

The KAPAO collaboration has designed, built and deployed a capable AO system at the Table Mountain Observatory 1-meter telescope, using Robo-AO control software from our collaborators at CIT. KAPAO is a dual-band optical and near-infrared MEMS DM AO system that has demonstrated substantial (Strehl >0.4) correction in both channels. The high-speed acquisition possible with both channels allowed the application of AO Lucky imaging techniques. The project is notable for the considerable undergraduate student contribution in virtually every aspect. The KAPAO program has a mature pipeline of students and now that the system shares the Cassegrain focus of the remotely operable telescope full-time, KAPAO is ready for regular operation.

\section{ACKNOWLEDGMENTS}

This material is based upon work supported by the National Science Foundation under Grant No. 0960343. Additional funding for aspects of this work were provided by the Pomona College Summer Undergraduate Research Program, the Rose Hills Foundation (PC student funding), the Newkirk student assistantship (SSU student funding) and the Mt. Cuba Astronomical Foundation which supported the SSU AO testbed. Further acknowledgement should be made to early work in MEMS AO by the Villages team led by Don Gavel at the UCO/Lick Observatory Laboratory for Adaptive Optics and for the early work on the CIT Camera project by Matthew Britton.

\section{REFERENCES}

[1] Baranec, C., Riddle, R., Ramaprakash, A. N., Law, N., Tendulkar, S., Kulkarni, S., Dekany, R., Bui, K., Davis, J., Burse, M., Das, H., Hildebrandt, S., Punnadi, S., and Smith, R., "Robo-AO: autonomous and replicable laser-adaptive-optics and science system," in [Society of Photo-Optical Instrumentation Engineers (SPIE) Conference Series], Society of Photo-Optical Instrumentation Engineers (SPIE) Conference Series 8447 (July 2012).

[2] Severson, S. A., Choi, P. I., Contreras, D. S., Gilbreth, B. N., Littleton, E., McGonigle, L. P., Morrison, W. A., Rudy, A. R., Wong, J. R., Xue, A., Spjut, E., Baranec, C., and Riddle, R., "KAPAO: a MEMSbased natural guide star adaptive optics system," in [Society of Photo-Optical Instrumentation Engineers (SPIE) Conference Series], Society of Photo-Optical Instrumentation Engineers (SPIE) Conference Series 8617 (Mar. 2013).

[3] Gavel, D., Severson, S., Bauman, B., Dillon, D., Reinig, M., Lockwood, C., Palmer, D., Morzinski, K., Ammons, M., Gates, E., and Grigsby, B., "Villages: an on-sky visible wavelength astronomy AO experiment using a MEMS deformable mirror," in [Society of Photo-Optical Instrumentation Engineers (SPIE) Conference Series], Society of Photo-Optical Instrumentation Engineers (SPIE) Conference Series 6888 (Mar. 2008).

[4] Gavel, D., Ammons, M., Bauman, B., Dillon, D., Gates, E., Grigsby, B., Johnson, J., Lockwood, C., Morzinski, K., Palmer, D., Reinig, M., and Severson, S., "Visible light laser guidestar experimental system (Villages): on-sky tests of new technologies for visible wavelength all-sky coverage adaptive optics systems," in [Society of Photo-Optical Instrumentation Engineers (SPIE) Conference Series], Society of Photo-Optical Instrumentation Engineers (SPIE) Conference Series 7015 (July 2008).

[5] Grigsby, B., Lockwood, C., Baumann, B., Gavel, D., Johnson, J., Ammons, S. M., Dillon, D., Morzinski, K., Reinig, M., Palmer, D., Severson, S., and Gates, E., "ViLLaGEs: opto-mechanical design of an onsky visible-light MEMS-based AO system," in [Society of Photo-Optical Instrumentation Engineers (SPIE) Conference Series], Society of Photo-Optical Instrumentation Engineers (SPIE) Conference Series $\mathbf{7 0 1 8}$ (July 2008).

[6] Morzinski, K., Johnson, L. C., Gavel, D. T., Grigsby, B., Dillon, D., Reinig, M., and Macintosh, B. A., "Performance of MEMS-based visible-light adaptive optics at Lick Observatory: closed- and open-loop control," in [Society of Photo-Optical Instrumentation Engineers (SPIE) Conference Series], Society of Photo-Optical Instrumentation Engineers (SPIE) Conference Series 7736 (July 2010).

[7] Baranec, C., Riddle, R., Law, N., Ramaprakash, A. N., Tendulkar, S., Bui, K., Burse, M., Chordia, P., Das, H., Dekany, R., Kulkarni, S., and Punnadi, S., "Robotic visible-light laser adaptive optics," in [Proceedings of the Third AO4ELT Conference], Esposito, S. and Fini, L., eds. (Dec. 2013). 
[8] Baranec, C., Riddle, R., Law, N. M., Ramaprakash, A. N., Tendulkar, S. P., Bui, K., Burse, M. P., Chordia, P., Das, H. K., Davis, J. T. C., Dekany, R. G., Kasliwal, M. M., Kulkarni, S. R., Morton, T. D., Ofek, E. O., and Punnadi, S., "Bringing the Visible Universe into Focus with Robo-AO," Journal of Vibration Engineering 72, 50021 (Feb. 2013).

[9] Riddle, R. L., Burse, M. P., Law, N. M., Tendulkar, S. P., Baranec, C., Rudy, A. R., Sitt, M., Arya, A., Papadopoulos, A., Ramaprakash, A. N., and Dekany, R. G., "The Robo-AO software: fully autonomous operation of a laser guide star adaptive optics and science system," in [Society of Photo-Optical Instrumentation Engineers (SPIE) Conference Series], Society of Photo-Optical Instrumentation Engineers (SPIE) Conference Series $\mathbf{8 4 4 7}$ (July 2012).

[10] Cornelissen, S. A., Hartzell, A. L., Stewart, J. B., Bifano, T. G., and Bierden, P. A., "MEMS deformable mirrors for astronomical adaptive optics," in [Society of Photo-Optical Instrumentation Engineers (SPIE) Conference Series], Society of Photo-Optical Instrumentation Engineers (SPIE) Conference Series 7736 (July 2010).

[11] Morzinski, K. M., Norton, A. P., Evans, J. W., Reza, L., Severson, S. A., Dillon, D., Reinig, M., Gavel, D. T., Cornelissen, S., Macintosh, B. A., and Max, C. E., "MEMS practice: from the lab to the telescope," in [Society of Photo-Optical Instrumentation Engineers (SPIE) Conference Series], Society of Photo-Optical Instrumentation Engineers (SPIE) Conference Series 8253 (Feb. 2012).

[12] Rigaut, F., "Yao, a monte-carlo simulation tool for adaptive optics (ao) systems," http://frigaut.github.com/yao/index.html.

[13] Fried, D. L., "Probability of getting a lucky short-exposure image through turbulence," Journal of the Optical Society of America (1917-1983) 68, 1651-1658 (Dec. 1978).

[14] Law, N. M., Mackay, C. D., and Baldwin, J. E., "Lucky imaging: high angular resolution imaging in the visible from the ground," 446, 739-745 (Feb. 2006).

[15] Severson, S., "Designing Effective Undergraduate Research Experiences," in [Learning from Inquiry in Practice], Hunter, Metevier, A. L., ed., Astronomical Society of the Pacific Conference Series 436, 449 (Dec. 2010). 